\title{
Depression in Stroke Rehabilitation
}

\author{
Joseph A. Schwartz, Nancy M. Speed, James A. Brunberg, Thomas L. Brewer, \\ Morton Brown, and John F. Greden
}

\begin{abstract}
Despite recent advances in understanding the pathophysiology of poststroke depression, major questions remain. They include the relative importance of lesion location and size and the confounding effects of time since stroke, age, prior history of depression, and cerebral atrophy. To evaluate these issues, we systematically assessed depressive features, functional status, and brain structure with computer tomography scans in 91 men undergoing stroke rehabilitation. Forty percent met DSM-III criteria for major depressive disorder. Mood disturbance was more severe for patients with right than with left hemisphere lesions, correlated with functional disability and lesion size, ani was associated with previous history of depression. Age, time since stroke, and atrophy did not correlate with mood. Depression is common in delayed stroke recovery, regardless of lesion location. Because there are no demographic or anatomic features that predict the absence of depression, depression screening should be part of the assessment of all patients undergoing stroke rehabilitation.
\end{abstract}

Key Words: Stroke, depression, organic affective disorder, CT scan

\section{Introduction}

It is estimated that within the United States, there are over 400,000 new stroke victims each year. Epidemiologic assessments indicate that poststroke depression is a commo. problem. In a community survey associated with the Framingham Study, Wolf et al (1990) found that depression was two times more common in patients 6 months after stroke than in an age-matched nonstroke control group. In a series of studies, Robinson et al $(1984 \mathrm{a}, 1984 \mathrm{~b})$ and Starkstein et al $(1987,1988 \mathrm{a}, 1988 \mathrm{~b}, 1988 \mathrm{c})$ explored strategies for overcoming difficulties in the clinical assessment of psychiatric symptoms in stroke patients, quantified severity of depressive symptoms, and investigated

From the Department of Psychiatry (JAS, TLB, JFG) and Radiology (JAB) and School of Public Health (MB), University of Michigan, and Psychiatry Service (NMS), Department of Veterans Affairs Medical Center, Ann Arbor, MI.

Address reprint requests to J. A. Schwartz, M.D., Department of Psychiatry, University of Michigan Hospital, 1500 E. Medical Center Drive, Ann Arbor, MI 48109/0020.

Received June 22, 1992; revised February 18, 1993. possible underlying mechanisms of mood disturbance. They established the utility of standard depression rating scales, confirmed that poststroke depressed patients often met DSM-III criteria for full-fledged major depressive disorder, and found a higher incidence of major depressive disorder with strokes proximate to the left frontal pole and involving the left caudate nucleus (Lipsey et al 1983; Robinson et al 1984a). They alse suggested that those with left posterior strokes or those involving the thalamus rarely developed depression (Starkstein et al 1988a).

However, consensus has not emerged with regard to these laterality issues. Specifically, Sinyor et al (1986b) failed to confirm any statistically significant association between left frontal lesion location and clinical depression. Both Sharpe et al (1990) and Egelko et al (1989) also failed to replicate a left-sided association; the latter study actually concluded that patients with right-sided strokes were more depressed (Egelko et al 1989). Reasons for these contrasting findings have not yet been thoroughly 
:xplored, but differences in methodology probably coniribute meaningfully.

One major source of variance might be the timing of r iood assessment following stroke. Wade et al (1987) followed nearly 1000 stroke patients for 1 year. Three weeks after stroke, patients with right brain injury were more depressed than those with left brain injury. All lateralized differences in the association with depression disappeared at 6 months poststroke. Only $50 \%$ of the sample was alive 1 year after the stroke, and early depression correlated with early death. Patients rated as "depressed" at 3 weeks had a 50\% higher moriality risk at 1 year. The mechanism by which this risk is conveyed is not known.

Questions about whether certain anatomic correlates predict subsequent depression should be clarified. This information would assist clinicians in early identification of patients at highest risk for depression, possibly help identify those stroke victims most likely to benefit from treatment interventions, and perhaps contribute to betier understanding of the etiology of depression. If successful treatment were instituted, rehabilitation might be shortened, costs reduced, quality of life improved, and unnecessary premature deaths prevented.

To investigate a number of these questions, we designed a prospective study of poststroke patients during the inpatisnt rehabilitation phase of their illness. The study design incorporated systematic assessment of depressive features, functional status, and brain imaging with computerized tomography (CT) scans. We found depression to be common following stroke; of greater severity in patients with large, right hemisphere lesions, correlated with a prior history of depression; and linked to functional disability. Patient age. time since stroke, and cerebral atrophy did not correlate with mood disorder.

\section{Methods}

\section{Population}

Patients for this study were selected from two populations: (1) consecutive admissions for stroke rehabilitation at the Minneapolis Veterans Administration Medical Center (VAMC) from July 1984 to January 1987; and (2) consecutive admissicns to the Ann Arbor VAMC Nu-sing Home Care Unit for stroke rehabilitation from July 1987 to December 1988.

One-hundred twenty-one patients were screened for study. Sixteen patients were excluded because CT scans were unavailable. Because of inadequate sample size, we also excluded the six women in this predominantly male sample. Four patients had brain stem lesions and were not included in the data analysis. Two CT scans were judged technically inadequate for the study, and these patients were excluded from the sample. Two patients were ex- cluded for severe receptive aphasia. Aphasia was assessed by the speech therapist. If any degree of receptive aphasia was noted by the speech therapist, the psychiatric evaluation included a series of yes/no questions of moderate complexity (i.e., "Do you put on your shoes before your socks? Is a short man shorter than a tall man?"). We excluded patients who scored less than $70 \%$ correct on this assessment. We included in the study four patients (two left, one right, and one bilateral) who scored $70 \%$ on the aphasia scale but could not, in the opinion of the psychiatrist, be rated on the Hamilton Rating Scale for Depression (HRSD). Ninety-orie patients met the criteria for the study.

\section{Psychological Assessment}

Two of us (JAS, NS) conducted a complete psychiatric history and jointly but independently assessed depression using the 24-item HRSD (Hamilton 1960). The Pearson correlation for the HRSD scores by the two investigators was $0.98(p<0.001)$. Averaged HRSD scores from both raters were used for analysis. DSM-III diagnosis of major depression was present or absent on the basis of consensus agreement of both interviewers.

We compiled an operational definition of "previous depression," consisting of acknowledgment of either (1) previous treatment of depression or (2) a 2-week period of significant aecline in funcion associated with an untreated depressed mood, unrelated to grief, or drug or alcohol use. In most cases, the previous history was confirmed by a family member.

\section{Assessment of Functional Status}

The Crichton Geriatric Rating Scale (Guy 1976) was used to measure the severity of physical impairment, from 11 (no impairment) to 55 (completely debilitated, disoriented, and bedridden.)

\section{CT Analysis}

All CT scans were assessed by a neuroradiologist (JB), who was unaware of the clinical state. Each stroke lesion was classified (1) as involving the left hemisphere only, right hemisphere only, or both (bilateral); or (2) by the presence or absence of involvement of the following brain structures in either hemisphere: frontal, temporal, parietal, or occipital ccrtex; internal capsule; and four subcortical nuclei (caudate, putamen, globus pallidus, and thalamus).

Measurement of stroke location and size, as reflected on the CT, was conducted using a Macintosh Computer with Pen Mac Planimeter Software and a backlit Numonics digitizing tablet. Measurements for each stroke lesion in- 
Table 1. Characteristics of the 91 Patients Studies

\begin{tabular}{|c|c|c|}
\hline & Niean $\pm S D$ & Range \\
\hline Age $(y r)$ & $66.0 \pm 9.2$ & $35-88$ \\
\hline HRSD score & $15.3 \pm 10.0$ & $0-38$ \\
\hline Crichton score & $22.9 \pm 8.1$ & $11-49$ \\
\hline Lesion area $(\%$ & $7 \pm 7$ & $0.1-32$ \\
\hline \multicolumn{3}{|l|}{ Race } \\
\hline White & & \\
\hline Nonwhite & & \\
\hline \multicolumn{3}{|l|}{ Marital status } \\
\hline Married and living together & & \\
\hline Single, separated, or widowed & & \\
\hline
\end{tabular}

${ }^{a}$ HDRS, Hamilton Rating Scale for Depression.

cluded mean anterior margin, mean posterior margin, and lesion area (Starkstein et al 1988c). Bicaudate diameter was measured as described previously by Gomori et al (1984) as an index of atrophy.

\section{Data Analysis}

All measures of variation are stated as standard deviation (SD). Correlation coefficients were used to link depression severity (HRSD) with other continuous measurements, whereas an ANOVA or $t$-tests were used to compare means for continuous measurements across groups. Groups were formed by dichotomizing the HRSD ( $<18$ versus $\geqslant 18$ ) by using a discrete variable, such as location of injury, or by using a combination of two such variables. Laterality was used to define three groups (bilateral, right, or left). Chi-square analysis was used to evaluate the association between dichotomous variables. The Mann-Whitney rank sum method was used to evaluate the effect of anatomic lesion location on HRSD scores.

\section{Results}

Sample characteristics are described in Table 1. For clinical reasons, the time duration from stroke to our assessment could not be rigorously controlled. The median time that had elapsed between the stroke and psychiatric evaluation was 2 months. Seventy-five percent of subjects were seen within the first 6 months after their strokes. Nine patients had their last confirmed stroke 2 or more years before admission; recent decline in functional status, however, suggested that extension of their previous stroke led to rehabilitative admissions, even though no new stroke was confirmed on CT scan. Because of these outliers, the average time since stroke was $7.7 \pm 15$ months (range 1-103).

\section{Clinical Measures}

We defined an HRSD score of $\geqslant 18$ as the dichotomous cutoff screen for depression. Using this cutoff score we describe two groups of patients: one with high HRSD ( $\geqslant$ 18 ) and one with low HRSD $(<18)$. Thirty-one (33\%) of our group had high HRSD scores. There were no differences in age $(t=0.04 ; \mathrm{df}=85 ; p=0.97)$ or time since stroke $(t=-0.75$; df $=85 ; p=0.45)$ for patients with high or low HRSD scores.

Thirty-six patients $(40 \%)$ of our sample met DSM-III criteria for major depressive episode. HRSD $\geqslant 18$ was strongly associated with DSM-III diagnosis of major depression in this sample $\left(\chi^{2}=46 ; \mathrm{df}=1 ; p<0.0001\right)$. HRSD scores predicted the presence or absence of major depression in nearly all cases with unilateral stroke. For patients with unilateral stroke, a cutoff of 18 correctly predicted the presence or absence of DSM-III depression in 51 of these 53 patients. In contrast, the cutoff of 18 was less predictive of the diagnosis of major depression in the subjects with bilateral stroke lesions. In the bilateral group, HRSD scores correctly predicted the presence or absence of DSM-III major depression in only 26 of the 34 patients. We found that 6 of the 34 bilateral stroke subjects had DSM-III depression and low HRSD. Two patients with high HRSD who were classified as not having DSM-III major depression also had bilateral lesions.

A previous history of depression was noted for 21 of the 81 patients for whom this could be reliably assessed. Fourteen of the 21 patients with a history of depression met DSM-III criteria for depression at the time of evaluation. A history of depression (Hx Depression) was strongly associated with poststroke depression $\left(x^{2}=7.89\right.$; $\mathrm{df}=$ $1 ; p=0.005)$. There was no difference in the frequency of previous depression in each of the lesion laterality categories (31\% left, $26 \%$ right, $21 \%$ bilateral).

\section{Depression and Anatomic Localization}

Figure 1 shows the proportions of depressed patients with lesions in each of the specific anatomic locations assessed in each hemisphere. Using the Mann-Whitney rank sum method, HRSD scores were significantly increased in those with occipital lesions (19.9 versus 14.0 for all other patients; $p=0.03$ ) and left internal capsule lesions (22.9 versus $10.3 ; p=0.015$ ) These findings are of uncertain significance because of the large number of comparisons (48) required for their discovery.

\section{Depression and Laterality}

Table 2 illustrates linkages between laterality and selected demographic, clinical, and CT variables. Chi-square analysis indicated that those with right-sided lesions were more 


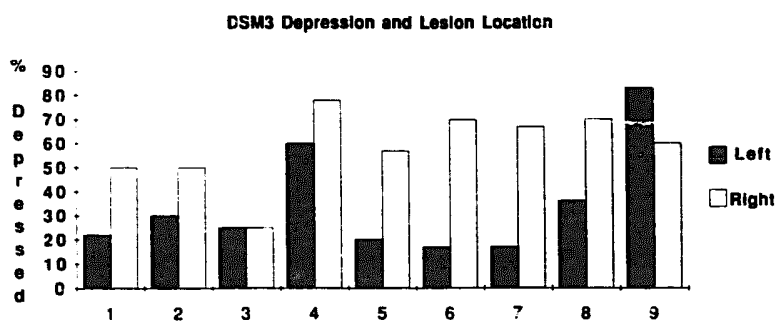

Figure 1. Frequency of depression for patients with stroke located in different brain regions: 1 , frontal lobe; 2 , temporal lobe; 3 , parietal lobe; 4 , occipital lobe; 5 , caudate nucleus; 6 , globus pallidus; 7 , putamen; 8 , tha'amus; 9 , internal capsule.

likely to have a high HRSD than those with left-sided or bilateral lesions $(p=0.03$ ). The proportions for meeting DSM-III criteria for major depression are similar, but their difference is marginally nonsignificant $(p=0.06$.)

Table 3 shows the result of linear regression analysis

Table 2. Patient Characteristics by Lesion Laterality ${ }^{a}$

\begin{tabular}{lccc}
\hline & $\begin{array}{c}\text { Right } \\
(n=28)\end{array}$ & $\begin{array}{c}\text { Left } \\
(n=28)\end{array}$ & $\begin{array}{c}\text { Bilateral } \\
(n=35)\end{array}$ \\
\hline Age (yr) & $63.8 \pm 9.3$ & $64.9 \pm 10.3$ & $68.7 \pm 7.5$ \\
HRSD score & $20.1 \pm 11.7$ & $13.2 \pm 8.7$ & $13.2 \pm 8.2$ \\
DSM-III MDD & $16(57 \%)$ & $7(25 \%)$ & $13(37 \%)$ \\
Hx depression (\%) & 26 & 31 & 21 \\
Lesion area (\%) & $10.0 \pm 7.4$ & $7 \pm 7$ & $4.2 \pm 5.1$ \\
Crichton score & $26.0 \pm 10.0$ & $22.0 \pm 6.4$ & $20.8 \pm 6.9$ \\
\hline
\end{tabular}

${ }^{a}$ Values are mean \pm SD; HRSD, Hamilton Rating Scale for Depression; MDD major depressive disorder.

Table 3. Predistion of HRSD by Lesion Location ${ }^{a}$

\begin{tabular}{cccccc}
\hline Variable & Average & SD & $F$ & $\begin{array}{c}p \\
\text { Value }\end{array}$ & $R^{2}$ \\
\hline $\begin{array}{c}\text { All subjects } \\
(n=87)\end{array}$ & & & & & \\
Anterior & 0.358 & 0.168 & 0.05 & NS & 0.001 \\
$\begin{array}{c}\text { Posterior } \\
\text { Bilateral }\end{array}$ & 0.631 & 0.207 & 7.12 & $<0.01$ & 0.077 \\
$(n=34)$ & & & & & \\
Anterior & 0.333 & 0.154 & 0.006 & NS & 0.00 \\
Posterior & 0.534 & 0.180 & 0.274 & NS & 0.008 \\
Left & & & & & \\
$(n=26)$ & & & & & \\
Anterior & 0.398 & 0.160 & 0.012 & NS & 0.000 \\
Posterior & 0.675 & 0.176 & 0.157 & NS & 0.007 \\
Right & & & & & \\
$(n=27)$ & & & & & \\
Anterior & 0.350 & 0.191 & 0.254 & NS & 0.010 \\
Posterior & 0.708 & 0.226 & 4.80 & $<0.05$ & 0.161 \\
\hline
\end{tabular}

${ }^{\circ}$ HRSD, Hamilton Rating Srale for Depression; NS, not significant.

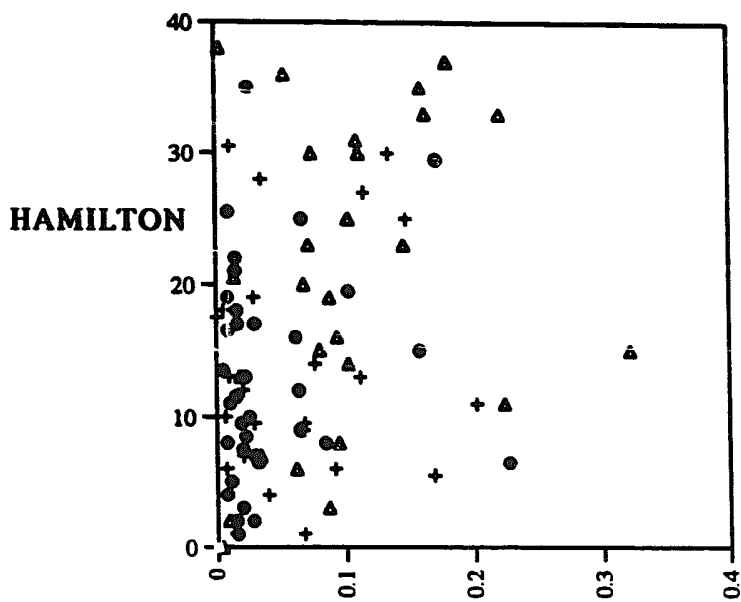

STROKE AREA

Figure 2. Relationship between HRSD scores and lesion area for the 91 subjects: $(0)$ bilateral; $(+)$ left; $(\Delta)$ right.

of the relationships between the linear anterior-posterior icsiuri iucation and HRSD scores. There was no significant relatinnship between the anterior margin of the lesion and HRSD for the group of 91 patients or for patients with bilateral, right, or left hemisphere lesions. The posterior margin of the lesion was significantly associated with HRSD for the whole sample and for those patients with rightsided stroke lesions but was nonsignificant for patients with bilateral or left-sided lesions.

\section{Depression and Lesion Characteristics}

The entire sample had an average lesion area of $7 \% \pm$ $7 \%$. The largest stroke lesions affected $32 \%$ of brain area, whereas the smallest measurable lesion involved $0.1 \%$ of brain area. Figure 2 shows the relationship between lesion area and HRSD scores for the entire sample. In all subjects there was a significant correlation between HRSD scores and lesion area $(r=0.28$; $\mathrm{df}=88 ; p<0.01)$. This relationship between lesion size and HRSD score was not seen in the subsets of laterality, perhaps because of smaller sample sizes.

\section{Depression and Functional Limitations}

For all subjects, severity of depression on the HRSD correlated sigrificantly with more impaired status, as assessed by the Crichton disability scale $(r=0.41$; $\mathrm{df}=89 ; p<$ 0.001 ). Significant correlations were also found for patients with right-sided lesions $(r=0.49$; $\mathrm{df}=23 ; p=$ 
$0.02)$ and those with left-sided lesions $(r=0.40$; $\mathrm{df}=$ $24 ; p=0.03$ ).

Crichton scores were significantly correlated with lesion area for the whole sample $(r=0.33$; $\mathrm{df}=89 ; p<0.01)$ as well as for all laterality subgroups. Crichton scores were significantly higher for patients with temporal $(27.7$ versus $20.0 ; p=0.0003$ ) and parietal (26.5 versus $20.6 ; p=$ 0.005 ) lesions.

\section{Depression and Linear Lesion Size and Atrophy}

The linear lesion size, derived by subtracting the anterior from the posterior lesion margin (Posterior - Anterior = Linear lesion size) was highly correlated with lesion area for patients with unilateral lesions $(r=0.93$; df $=54$; $p<0.001$ ). Linear lesion size correlated with HRSD and Crichton scores in a nearly identical manner to lesion area. Bicaudate diameter as a measure of atrophy did not correlate significantly with $\mathrm{HRSD}(r=0.06$; $\mathrm{df}=1 / 85$; $p=0.59$ ).

\section{Discussion}

There are three important themes in poststroke mood research. The first addresses the questions of how many poststroke patients have depression and what is the severity of the syndromes. The second is whether there are focal neuroanatomic injuries that lead to depression and whether this knowledge might aid us in understanding the neurology of emotion. The third theme relates to clinical outcome and addresses the question of whether early identification of high-risk subgroups could lead to prevention of psychiatric comorbidity or improve the general outcome of stroke rehabilitation.

With regard to the first and second themes, $33 \%$ of male poststroke patients had significant depression, as measured by HRSD. Forty percent met DSM-III criteria for major depression. HRSD scores were higher for patients with large lesions, especially those which involved posterior structures. Patients with right-sided lesions appeared more depressed than those with left-sided lesions. The overall impression gained from this study is that the risk of depression was high for all patients, regardless of lesion location. With regard to severity, we found a significant relationship between functional impairment and depressive symptoms. Large stroke lesions caused the most functional disability and the most severe depression. We also observed this relationship between large stroke lesions and depression severity using SPECT single-photon emission CT (SPECT) with ${ }^{99}$ Tc-hexamethylpropyleneamine oxime (Schwartz et al 1990). Sinyor et al (1986a) and Robinson et al (1984b) similarly noted a correlation be- tween functional impairment and depression in stroke patients.

With regard to the second theme, our findings of higher rates of depression in right-sided brain injury are consistent with several previous reports. Lishman's (1968) large sample of patients with penetrating head injury exhibited a more frequent occurrence of affective disorder in patients with right-sided lesions. Dam et al (1989) report that stroke patients with right-hemisphere lesions had higher HRSD scores than those with left-hemisphere lesions. In Egelko et al's (1989) study of inpatients undergoing rehabilitation, those with right-hemisphere lesions appeared more depressed than those with left-hemisphere lesions.

For patients with right-hemisphere stroke, posterior lesions were associated with depression. This finding is consistent with observations of both Robinson et al (1984a) and Sinyor et al (1986a). In a study of 42 right-hemisphere stroke patients, Finset et al (1989) also found a significant correlation between deep posterior lesions and global assessment of depression.

Our data do not confirm the prior finding of a relationship between depression and left-hemisphere or left frontal lesions reported by Robinson et al (1984a). This discrepancy probably reflects methodological differences. In Robinson's sample, $50 \%$ of the patients had left-hemisphere strokes (versus $30 \%$ in ours), and $25 \%$ or more of their patients did not have CT-visible lesions. This suggests that average lesions were larger in our sample. Our sample appears to be more depressed than the group seen by Robinson. Another difference was the time elapsed between the stroke and the initial assessment of depression. For example, Robinson and colleagues conducted assessments of subjects on 7-21 days after the stroke (Starkstein et al $1987,1988 b, 1988 c)$. Anatomic location of stroke, as defined by Robinson and colleagues, may thus have a greater influence on mood earlier in the course of the stroke. At later time points, such as those used in our sample, and usually after rehabilitation had begun, the total volume of brain tissue damaged and the degree of functional impairment may becorne more important predictors of depression than lesion location.

A third theme pertains to optimal ways to assess patients and plan early interventions. Although CT scanning techniques are helpful for diagnosing stroke, we believe that they provide limited insight into poststroke depression. Cerebral blood flow imaging with SPECT can be helpful in evaluating the extent of the brain injury, especially in cases where physical disability seems disproportionate to CT findings. SPECT is more sensitive to ischemia than $\mathrm{CT}$ and may be useful in future studies of the association between brain injury and mood disorders.

Because there are no demographic or anatomic lesions 
that predict the absence of depression, depression screening should be part of the assessment of all patients undergoing stroke rehabilitation. All subgroups appear to have high rates of depression. Patients with a previous history of depression and those with large stroke lesions should be routinely assessed by the consulting psychiatrist. The development of improved and detailed functional assessment tools may soon allow the investigation of the changes in recovery rates associated with treatment of mood disturbances in stroke patients.

In summary, the best model of poststroke depression that we could construct would involve both lesion location and time. Left frontal damage may cause a depression that has an early appearance and is associated with either re-

\section{References}

Dam H, Pederson HE, Ahlgren P (1989): Depression among patients with stroke. Acta Psychiatr Scand 80:118-124.

Egelko S, Simon D, Riley E, Gordon W (1989): First year after stroke: Tracking cognitive and affective deficits. Arch Phys Med Rehabil 70:297-302.

Finset A, Goffeng L, Landro I, Haakonsen M (1989): Depressed mood and intra-hemispheric location of lesion in right hemisphere stroke patients. Scand J Rehab Med 21:1-6.

Gomori JM, Steiner I, Melamed E, Cooper G (1984): The assessment of changes in brain volume using combined linear measurements: A CT-scan study. Neuroradiology 26:21-24.

Guy W (1976): ECDEU: Assessment Manual for Psychopharmacology. Washington, DC: US1 DHEW.

Hamilton M (1960): A rating scale for depression. J Neurosurg Psychiatry 23:56-62.

Lipsey J, Robinson R, Pearlson G (1983): Mood change following bilateral hemisphere brain injury. Br J Psychiatry 143:266273.

Lishman W (1968): Location of brain damage in relation to psychiatric disability after heac̉ injury. Br J Psychiatry 114:373410.

Robinson R, Kubos K, Starr L (1984a): Mood disorders in stroke patients-importance of location of lesion. Brain 107:81-93.

Robinson R, Starr L, Lipsey J (1984b): A two-year longitudinal study of poststroke mood disorders: Dynamic changes in associated variables over the first six months of follow-up. Stroke 15(3):510-517

Schwartz JA; Speed NM, Mountz JM, Gross MD, Modell J, Kuhl DE (1990): 99-Tc-hexamethylpropyleneamine oxime single photon emission CT in poststroke depression. Am J Psychia:ry 147:242-244. covery or death. The patient with a large right posterior lesion may develop depression later in the recovery process. Thus, poststroke evaluations in a short-term medical setting will see a high depression rate in patients with left frontal stroke. Studies conducted in an inpatient rehabilitation setting would show higher rates of depression in patients with large right posterior lesions. Clearly, early interventions would be desirable if they prevented subsequent depression, lessened its severity, shortened its course, and were safe. Treatment trials will be needed to address these important outcome questions.

This work was supported in part by VAMH-RCS grant 10-0159 and NIM Biomedical Research Suppon Grant RR-05383.

Sharpe M, Hawton K, House A, et al (1990): Mood disorders in long-term survivors of stroke: Associations with brain lesion location and volume. Psychol Med 20:815-828.

Sinyor D, Amato P, Kaloupek D (1986a): Relationship to functional impairment, coping, strategies, and rehabilitation outcome. Stroke 17:1102-1107.

Sinyor D, Jacques P, Kaloupek D (1986b): Poststroke depression and lesion location: An attempted replication. Brain 109:537546.

Starkstein S, Robinson R, Price T (1987): Comparison of cortical and subcortical lesions in the production of poststroke mood disorders. Brain 110:1045-1059.

Starkstein S, Robinson R, Bertheir M, Parikh R, Price T (1988a): Differential mood changes following basal gaglia vs thalamic lesion. Arch Neurol 45:725-730.

Starkstein S, Robinson R, Bertheir M, Price T (1988b): Depressive Disorders following posterior circulation as compared with middle cerebral artery infarcts. Brain 111:375387.

Starkstein S, Robinson R, Price T (1988c): Comparison of patients with and without poststroke major depression matched for size and location of lesion. Arch Gen Psychiatry 45:247252.

Wade D T, Legh-Smith J, Hewer R A (1987): Depressed mood after stroke: A community study of its frequency. Br J Psychiatry 151:200-205.

Wolf PA, Bachman DL, Kelly-Hayes M, et al (1990): Stroke and depression in the community: The Framingham stuvy. Neurology 40(1):416. 